Canad. Math. Bull. Vol. 22 (1), 1979

\title{
SELF-CONVERSE TOURNAMENTS
}

BY

W. J. R. EPLETT

Let $T_{n}$ denote a tournament with vertices labelled $1, \ldots, n$. Any undefined terms can be found in [5]. The converse of $T_{n}$ is the tournament $T_{n}^{\prime}$ obtained by reversing the orientation of all the $\operatorname{arcs}$ in $T_{n}$. A tournament is called self-converse (s.c.) if $T_{n} \cong T_{n}^{\prime}$. The transitive tournaments are examples of s.c. tournaments. In this paper we provide a structural characterization of s.c. tournaments and we also characterize the score vectors of s.c. tournaments.

1. The structure of S.C. tournaments. An automorphism of $T_{n}$ is a permutation of the vertices that preserves the orientation of all $\operatorname{arcs}$ of $T_{n}$; an anti-automorphism is a permutation that reverses all arcs. The group of all automorphisms of $T_{n}$ will be denoted by $G=G\left(T_{n}\right)$ and the group of all automorphisms and anti-automorphisms of $T_{n}$ will be denoted by $H=H\left(T_{n}\right)$. Clearly, $T_{n}$ is s.c. if and only if $G$ is a proper subgroup of $H$. In particular, if $T_{n}$ is s.c., then $H$ is a group of order $2 m, m$ an odd integer, so there must be a self-inverse anti-automorphism (with one fixed vertex when $n$ is odd). Hence the vertices of an s.c. tournament $T_{n}$ may be labelled so that the permutation $\gamma: i \rightarrow n+1-i$ for $1 \leq i \leq n$ is an anti-automorphism.

Notice that not all anti-automorphisms of an s.c. tournament are necessarily self-inverse. For instance, the tournament on $p$ vertices, where $p$ is a prime of the form $4 l+3$, in which $i$ dominates $j$ if and only if $j-i$ is a quadratic residue modulo $p$, has an anti-automorphism of order $p-1$.

Suppose that $T_{n}$ denotes any s.c. tournament with $n=2 m+1$ vertices, labelled as above with respect to some self-inverse anti-automorphism $\gamma$. The vertex $m+1$ is fixed under $\gamma$ and has score $m$. If $A=A\left(T_{n}\right)$ and $C=C\left(T_{n}\right)$ denote the subtournaments of $T_{n}$ determined by the $m$ vertices that dominate $m+1$ and the $m$ vertices that are dominated by $m+1$, then clearly $C \cong A^{\prime}$. Notice that if $i$ is a vertex of $A$, then $n+1-i$ is a vertex of $C$, while the arc $(i, n+1-j)$ is in $T_{n}$ if and only if $(j, n+1-i)$ is in $T_{n}$. Define $B=B\left(T_{n}\right)$ to be the undirected graph whose $m$ vertices have the same labels as the vertices of $A$ and in which an edge joins $i$ and $j$ if and only if the arc $(i, n+1-j)$ is in $T_{n}$ (this includes the possibility that $i=j$ ). The tournament $A$ and the undirected graph $B$ together determine the s.c. tournament $T_{n}$. This pair will be called a representation of $T_{n}$.

Received by the editors November 8, 1976 and in revised form, December 1st, 1977 and April 18, 1978. 
Representations of the s.c. tournaments $V$ and $W$ are isomorphic if there exists a mapping $\phi$ so that both $A(V) \cong A(W)$ and $B(V) \cong B(W)$ under $\phi$. If all the representations of an s.c. tournament $T_{n}$ obtained from the different self-inverse anti-automorphisms are isomorphic, $T_{n}$ may be said to have a unique representation.

Proposition. (a) The s.c. tournament $T_{n}$ has a unique representation. (b) Two s.c. tournaments are isomorphic if and only if their representations are isomorphic.

Proof. To prove (a) we show that if $\gamma$ is a given self-inverse antiautomorphism, then any other self-inverse anti-automorphism may be written as $g^{-1} \circ \gamma \circ g$ for some $g \in G$. This will prove (a) because of the way conjugation preserves the cycle structure of a permutation [2, p. 54].

Since $H$ has order $2 m, m$ odd, any two Sylow 2-subgroups are conjugate in $H$ and so any self-inverse anti-automorphism may be written as $h^{-1} \circ \gamma \circ h$ for some $h$ in $H$. The required result follows from this because $h \in H \backslash G$ may be written as $\gamma \circ \mathrm{g}$ for some $\mathrm{g}$ in $\mathrm{G}$. One may now speak of the representation of $T_{n}$.

Let $V$ and $W$ be isomorphic under the mapping $\Psi$ from the vertices of $V$ to the vertices of $W$. If $\gamma$ is a self-inverse anti-automorphism of $V$, then $\Psi \circ \gamma \circ \Psi^{-1}$ is a self-inverse anti-automorphism of $W$ and the representation of $W$ obtained from this anti-automorphism is clearly isomorphic to the representation of $V$ obtained from $\gamma$. This proves one part of (b); the other part is straightforward.

This proposition may be used to count the number of unlabelled s.c. tournaments in the following way.

COROLlaRy 1. If $s(n)$ denotes the number of unlabelled s.c. tournaments on $n$ vertices, then for $m \geq 1$

$$
s(2 m+1)=\frac{1}{m !} \sum_{(j)} \frac{m !}{\Pi k^{j_{k}} j_{k} !} 2^{t(j)},
$$

where

$$
t(j)=\sum_{r, t=1}^{m}(r, t) j_{r} j_{t}
$$

(here $(r, t)$ denotes the g.c.d. of $r$ and $t$ ) and the sum is over all partitions $m=1$. $j_{1}+2 . j_{2}+3 . j_{3}+\ldots$, where the $j_{i}$ are non-negative integers and $j_{i}=0$ whenever $i$ is even.

Proof. The proof consists of using the proposition and results contained in chapters 4 and 5 of [3], together with an application of Burnside's lemma. The details are routine and we omit them.

This is perhaps a more straightforward way of obtaining $s(2 m+1)$ than that suggested in [3], indicating why partitions of $m$ rather than $2 m+1$ are 
TABLE. The number of s.c. tournaments

\begin{tabular}{rr}
\hline$n$ & $s(n)$ \\
5 & 8 \\
6 & 12 \\
7 & 88 \\
8 & 176 \\
9 & 2752 \\
10 & 8784 \\
11 & 279968 \\
\hline
\end{tabular}

appropriate in the summation. Some of the numbers $s(n)$ are displayed in the accompanying table. For the case when $n$ is even, the formula on p. 156 of [3] was used to compute $s(n)$. Notice that that formula can be written in terms of a summation over partitions of $n / 2$ to simplify computation.

A curious feature of this representation for s.c. tournaments with an odd number of vertices is that there seems to be no really satisfactory way of extending it to s.c. tournaments with an even number of vertices. For any possible representation to be unique it must be preserved in a natural way by automorphisms of the tournament. In the case where the tournament had an odd number of vertices we were able to use the fixed point to do this.

2. The score vectors of S.C. tournaments. In this section we obtain necessary and sufficient conditions for a sequence of $n$ non-negative integers to constitute the scores of some s.c. tournament on $n$ vertices.

Landau [4] proved that a necessary and sufficient condition for the nonnegative integers $s_{1} \leq \cdots \leq s_{n}$ to be the scores of a tournament is given by

$$
\sum_{i=1}^{k} s_{i} \geq\left(\begin{array}{l}
k \\
2
\end{array}\right),
$$

for $1 \leq k \leq n$ with equality when $k=n$. This will be called condition I. From observations in section 1 it follows that to be the scores of an s.c. tournament these integers must also satisfy $s_{i}+s_{n+1-i}=n-1$, for $1 \leq i \leq n$. This will be called condition II. Notice that if $s_{1} \leq \cdots \leq s_{n}$ satisfy II and

$$
\sum_{i=1}^{k} s_{i} \geq\left(\begin{array}{l}
k \\
2
\end{array}\right)
$$

for $1 \leq k \leq[n / 2]$, then $\mathrm{I}$ is automatically satisfied. This enables us to work with only the first $[n / 2]$ integers. The proof of the following theorem was suggested by [1].

THEOREM. The non-negative integers $s_{1} \leq \cdots \leq s_{n}$ are the scores of an s.c. tournament if and only if they satisfy I and II. 
Proof. Suppose the integers $s_{1} \leq \cdots \leq s_{n}$ satisfy I and II. Consider first the case when $n=2 m+1$ where, as we may suppose, $m \geq 2$. Define the functions $Q(k)$ and $R(k)$ as follows:

$$
R(k)=\sum_{i=1}^{k} s_{i}-\left(\begin{array}{l}
k \\
2
\end{array}\right)
$$

and

$$
Q(k)=\sum_{i=1}^{k}\left(s_{i}-1\right)-\left(\begin{array}{l}
k \\
2
\end{array}\right) .
$$

Then $R(k) \geq 0$ for $i \leq k \leq m-1$ and we assume that

$$
\min \{Q(k): 1 \leq k \leq m-1\}=-u
$$

for some positive integer $u$. (The possibility that $Q(k) \geq 0$ for $1 \leq k \leq m-1$ will be disposed of later). Let

$$
\min \{k: Q(k)=-u\}=j_{u}
$$

Since

$$
Q(k)=R(k)-k \geq-k,
$$

it follows that $1 \leq u \leq j_{u} \leq m-1$. Define $j_{1}, \ldots, j_{u-1}$ as follows: $j_{i}$ is the smallest integer $t$ such that $1 \leq t<j_{i+1}$ and $Q(t)=-i$, if such an integer exists; otherwise $j_{i}=j_{i+1}-1$. It is not difficult to see, appealing to $\left(^{*}\right)$, that $1 \leq j_{1}<\cdots<j_{u} \leq$ $m-1$. Let $J=\left\{j_{i}: 1 \leq i \leq u\right\}$.

Consider the following $2 m-1$ modified scores $\left(s_{1}^{\prime}, \ldots, s_{m-1}^{\prime}, s_{m+1}^{\prime}\right.$, $\left.s_{m+3}^{\prime}, \ldots, s_{2 m+1}^{\prime}\right)$ where $s_{i}^{\prime}=s_{i}$ and $s_{2 m+2-i}^{\prime}=s_{2 m+2-i}-2$ if $i \in J$ and $s_{i}^{\prime}=s_{i}-1$ for the remaining relevant values of $i$. If $Q(k) \geq 0$ for $1 \leq k \leq m-1$, then only the second part of the definition applies. These scores satisfy conditions I and II; to show that $s_{1}^{\prime} \leq \cdots \leq s_{m-1}^{\prime} \leq s_{m+1}^{\prime} \leq \cdots \leq s_{2 m+1}^{\prime}$, it suffices to consider the cases where $s_{k}^{\prime}=s_{k}$ and $s_{k+1}^{\prime}=s_{k+1}-1$. In such cases it must be that $k=j_{i}$ for some $i$ and $k+1<j_{i+1}$. Consequently, $s_{k}-k=Q(k)-Q(k-1)<0$ and $s_{k+1}-(k+1)=$ $Q(k+1)-Q(k) \geq 0$, from the definition of $j_{i}$. Hence $s_{k}<s_{k+1}$ and the required inequality certainly holds. We may suppose, therefore, as an induction hypothesis that there exists an s.c. tournament $T_{2 m-1}$ with $2 m-1$ vertices labelled $1, \ldots, m-1, m+1, m+3, \ldots, 2 m+1$ with scores $s_{1}^{\prime}, \ldots, s_{m-1}^{\prime}, s_{m+1}^{\prime}$, $s_{m+3}^{\prime}, \ldots, s_{2 m+1}^{\prime}$. We may further suppose that the mapping $\gamma: i \rightarrow 2 m+2-i$, for $1 \leq i \leq m-1$ and $i=m+1$, defines an anti-automorphism.

Now we propose to join two vertices labelled $m$ and $m+2$ to $T_{2 m-1}$ and orient arcs joining these vertices to each other and to the vertices of $T_{2 m-1}$ in such a way that the resulting tournament $T_{2 m+1}$ is an s.c. tournament with scores $s_{1}, \ldots, s_{2 m+1}$. Orient the arcs as follows: $\left(m, j_{i}\right),\left(m+2, j_{i}\right),(2 m+2-$ $\left.j_{i}, m+2\right),\left(2 m+2-j_{i}, m\right)$ for $1 \leq i \leq u$. (This takes care of all arcs incident with the vertices $j_{i}$ and $2 m+2-j_{i}$ and these vertices clearly have the required score 
now. If $Q(k) \geq 0$ for $1 \leq k \leq m-1$, then this step does not apply). Furthermore, vertex $m+1$ is dominated by $m$ and dominates $m+2$.

Now suppose that $s_{m}=u+2 v+1+\delta$ where $\delta$ equals 0 or 1 . (Notice that $2 v+\delta \geq 0$; for writing $j$ for $j_{u}$, then

$$
\begin{aligned}
0 \leq R(j+1) & =R(j)+s_{j+1}-(j+1)=Q(j)+s_{j+1}-1 \\
& \leq-u+s_{m}-1=2 v+\delta .
\end{aligned}
$$

Also, the fact that $s_{m} \leq s_{m+1}=m$ implies that $v \leq 2 v \leq m+1-u-\delta$.)

To continue the definition of $T_{n}$ orient the arcs as follows: $(m, i),(m, 2 m+$ $2-i),(i, m+2),(2 m+2-i, m+2)$ for $i$ running through the first $v$ positive integers not in $J,(i, m),(2 m+2-i, m),(m+2, i),(m+2,2 m+2-i)$ for the next $m-1-u-v$ positive integers not in $J$. Finally $m$ dominates or is dominated by $m+2$ according as $\delta$ equals 1 or 0 . It is easy to verify that the tournament $T_{n}$ defined in this way is indeed an s.c. tournament with scores $s_{1}, \ldots, s_{n}$, as required. This suffices to prove the theorem for odd values of $n$ by induction. The proof for even $n$ is similar and will be omitted.

ACKnowledgement. Special gratitude is due to Professor J. W. Moon, many of whose suggestions have been incorporated in the revised version of this paper. The assistance of the referee and Dr. J. N. Ridley is also gratefully acknowledged.

\section{REFERENCES}

1. A. Brauer, I. C. Gentry and K. Shaw, A new proof of a theorem by H. G. Landau on tournament matrices, J. Combinatorial Theory 5 (1968), 289-292.

2. M. Hall, The Theory of Groups, Macmillan, New York, 1970.

3. F. Harary and E. M. Palmer, Graphical Enumeration, Academic Press, New York, 1973.

4. H. G. Landau, On dominance relations and the structure of animal societies III: the conditions for a score structure, Bull. Math. Biophys, 15 (1953), 143-148.

5. J. W. Moon, Topics on Tournaments, Holt, Rinehart and Winston, New York, 1968.

DEPARTMENT OF MATHEMATICS,

THE UNIVERSITY OF REAdING,

WHITEKNIGHTS,

REAdING RG6 2AX

ENGLAND 